PROCEEDINGS OF THE

AMERICAN MATHEMATICAL SOCIETY

Volume 126, Number 5, May 1998, Pages 1527-1530

S $0002-9939(98) 04179-3$

\title{
VOLUME OF INTERSECTIONS AND SECTIONS OF THE UNIT BALL OF $\ell_{p}^{n}$
}

\author{
MICHAEL SCHMUCKENSCHLÄGER
}

(Communicated by Dale Alspach)

\begin{abstract}
An asymptotic formula for the volume of the intersection of a suitable multiple of the unit ball of $\ell_{p}^{n}$ and the cube $[-1 / 2,1 / 2]^{n}$ will be proved. We also show that the isotropic constant of the unit ball of $\ell_{n}^{p}, 1 \leq p \leq 2$, is bounded by $1 / \sqrt{12}$.
\end{abstract}

\section{INTRODUCTION AND NOTATION}

Let $r(n, p) B_{p}^{n}$ be the multiple of the unit ball $B_{p}^{n}$ of $\ell_{p}^{n}$ such that

$$
\operatorname{Vol}_{n}\left(r(n, p) B_{p}^{n}\right)=1 .
$$

In [SS] the following theorem is proved:

Theorem 1.1. For all $0<p \leq \infty$ and all $0<q<\infty$ we have

$$
\lim _{n \rightarrow \infty} \operatorname{Vol}_{n}\left(r(n, p) B_{p}^{n} \cap \operatorname{t.r}(n, q) B_{q}^{n}\right)= \begin{cases}0 & \text { if } t A(p, q)<1 \\ 1 & \text { if } t A(p, q)>1\end{cases}
$$

where

$$
A(p, q)= \begin{cases}\frac{e^{1 / p} \Gamma\left(1+\frac{1}{p}\right)^{1+1 / q} p^{1 / q}}{e^{1 / q} \Gamma\left(1+\frac{1}{q}\right) \Gamma\left(\frac{1+q}{p}\right)^{1 / q} q^{1 / q}} & \text { if } p<\infty, \\ \frac{(1+q)^{1 / q}}{e^{1 / q} \Gamma\left(1+\frac{1}{q}\right) q^{1 / q}} & \text { if } p=\infty .\end{cases}
$$

Also, the following problem was posed: What is the asymptotic behavior of

$$
\operatorname{Vol}_{n}\left(r(n, p) B_{p}^{n} \cap \operatorname{t.r}(n, q) B_{q}^{n}\right)
$$

for $t=A(p, q)^{-1}$ ? In section 2 it will be proved that in the case $p=\infty$ this limit equals $\frac{1}{2}$ - the case $p=\infty$ and $q=1$ has also been solved by B. Weißbach.

In section 3 we consider a different problem: Let $E$ be a subspace of $\mathbf{R}^{n}$ of codimension $k$. M. Meyer and A. Pajor (cf. $[\mathrm{MeP}]$ ) proved that for all $p \geq 2$ and $p=1: \operatorname{Vol}_{n-k}\left(E \cap r(n, p) B_{p}^{n}\right) \geq 1$. We will prove this inequality in the case $k=1$ and $1<p<2$.

Received by the editors June 14, 1996 and, in revised form, October 14, 1996.

1991 Mathematics Subject Classification. Primary 52A20.

The author was supported in part by BSF and Erwin Schrödinger Auslandsstipendium J0630, J0804.

(C) 1998 American Mathematical Society 


\section{IntERSECTION With THE CUBE}

Let $0<q<\infty$ and $t=A(\infty, q)^{-1}$. Define $a(n, q)$ by the equation

$$
a(n, q) \frac{1}{2(1+q)^{1 / q}}=\frac{r(n, q)}{n^{1 / q} A(\infty, q)} .
$$

Using Stirling's formula it is easily checked that

$$
a(n, q)=1+\frac{q \log n}{2 n}+O\left(\frac{1}{n}\right) .
$$

Now, let $X$ be uniformly distributed on the interval $\left[-\frac{1}{2}, \frac{1}{2}\right]$ and let $X_{j}, j=1, \ldots, n$, be independent copies of $X$. Then

$$
\operatorname{Vol}_{n}\left(\left[-\frac{1}{2}, \frac{1}{2}\right]^{n} \cap \operatorname{tr}(n, p) B_{p}^{n}\right)=1-\mathbf{P}\left(\left(\frac{1}{n} \sum_{j=1}^{n}\left|X_{j}\right|^{q}\right)^{1 / q}>\|X\|_{q} a(n, q)\right) .
$$

Theorem 2.1. For all $0<q<\infty$ we have:

$$
\lim _{n \rightarrow \infty} \operatorname{Vol}_{n}\left(\left[-\frac{1}{2}, \frac{1}{2}\right]^{n} \cap \frac{r(n, q)}{A(\infty, q)} B_{q}^{n}\right)=\frac{1}{2} .
$$

Proof. By (1) and simple algebra we conclude that the probability in (2) is given by

$$
\mathbf{P}\left(\frac{1}{\sqrt{n}}\left(\sum_{j=1}^{n}\left|X_{j}\right|^{q}-\mathbf{E}|x|^{q}\right)>\|X\|_{q}^{q}\left(\frac{q^{2} \log n}{2 n}+O\left(\frac{1}{n}\right)\right) \sqrt{n}\right) .
$$

A version of the Berry-Esseen Theorem (cf. e.g. [C, p. 225]) states that if $Y_{j}$, $j=1, \ldots, n$, is an i.i.d. sequence of random variables such that $\mathbf{E} Y=0$ and $\|Y\|_{3}<\infty$, then there exists an absolute constant $C$ such that for all $s \in \mathbf{R}$ :

$$
\left|\mathbf{P}\left(\frac{1}{\|Y\|_{2} \sqrt{n}} \sum_{j=1}^{n} Y_{j}<s\right)-\frac{1}{\sqrt{2 \pi}} \int_{-\infty}^{s} e^{-1 / 2 x^{2}} d x\right| \leq C\left(\frac{\|Y\|_{3}}{\|Y\|_{2}}\right)^{2} \frac{1}{\sqrt{n}} .
$$

Applying this theorem to $Y=|X|^{q}-\mathbf{E}|X|^{q}$ and

$$
s=\frac{\|X\|_{q}^{q}}{\|Y\|_{2}}\left(\frac{q^{2} \log n}{2 \sqrt{n}}+O\left(\frac{1}{\sqrt{n}}\right)\right)=O\left(\frac{\log n}{\sqrt{n}}\right)
$$

we get:

$$
\left|\mathbf{P}\left(\left(\frac{1}{n} \sum_{j=1}^{n}\left|X_{j}\right|^{q}\right)^{1 / q}>\|X\|_{q} a(n, q)\right)-\frac{1}{2}\right| \leq C_{1} \frac{\log n}{\sqrt{n}}+C \frac{1}{\sqrt{n}},
$$

which proves the theorem. 


\section{Central sections of $B_{p}^{n}$}

Suppose $B$ is a convex symmetric body in $\mathbf{R}^{n}$ with $\operatorname{Vol}_{n}(B)=1$. It is well-known that there exists an affine image $\widetilde{B}$ of $B$ such that the function

$$
x \mapsto \int_{\widetilde{B}}\langle x, y\rangle^{2} d y
$$

is constant on $S^{n-1}$. This constant is called the isotropic constant of $B$ and is denoted by $L_{B}$. We also say that $\widetilde{B}$ is in isotropic position. It is easy to see that if the standard basis of $\mathbf{R}^{n}$ is a 1-symmetric basis of $B$, then $B$ is in isotropic position. Let $1 \leq p \leq 2$. Then

$$
L_{B_{p}^{n}}^{2}=\int_{r(n, p) B_{p}^{n}} x_{1}^{2} d x
$$

where as above $r(n, p)=\operatorname{Vol}_{n}\left(B_{p}^{n}\right)^{-1 / n}$. A direct computation yields:

$$
L_{B_{p}^{n}}^{2}=\frac{\Gamma\left(1+\frac{3}{p}\right) \Gamma\left(1+\frac{n}{p}\right)^{1+2 / n}}{12 \Gamma\left(1+\frac{n+2}{p}\right) \Gamma\left(1+\frac{1}{p}\right)^{3}} .
$$

Let $H$ be a hyperplane containing the origin. A well known result (cf. e.g. [B1]) states that:

$$
\operatorname{Vol}_{n-1}\left(r(n, p) B_{p}^{n} \cap H\right) L_{B_{p}^{n}} \geq \frac{1}{\sqrt{12}}
$$

In order to prove $\operatorname{Vol}_{n-1}\left(\alpha B_{p}^{n} \cap H\right) \geq 1$ it is enough to prove the following inequality:

$$
\frac{\Gamma\left(1+\frac{n}{p}\right)^{1+2 / n}}{\Gamma\left(1+\frac{n+2}{p}\right)} \leq \frac{\Gamma\left(1+\frac{1}{p}\right)^{3}}{\Gamma\left(1+\frac{3}{p}\right)} .
$$

By Stirling's formula we have:

$$
\Gamma(1+z)=\sqrt{2 \pi}(1+z)^{z+\frac{1}{2}} e^{-1-z} \exp (\gamma(z))
$$

where $\gamma$ is a decreasing function on the interval $[0, \infty)$ satisfying $0<\gamma(z)<$ $(12(z+1))^{-1}$. Putting $x=p^{-1}$ the inequality (4) can be written equivalently:

$$
\begin{aligned}
& (2 \pi(1+n x))^{1 / n}\left(\frac{1+n x}{1+(n+2) x}\right)^{1 / 2+(n+2) x} e^{1 / n+(1+2 / n) \gamma(n x)-\gamma((n+2) x)} \\
& \quad \leq 2 \pi(1+x)\left(\frac{1+x}{1+3 x}\right)^{1 / 2+3 x} e^{1+3 \gamma(x)-\gamma(3 x)}
\end{aligned}
$$

Putting $z_{n}=\frac{2 x}{1+(n+2) x}$ we get

$$
\begin{aligned}
\log \left(\frac{1+n x}{1+(n+2) x}\right)^{1 / 2+(n+2) x}-\log \left(\frac{1+x}{1+3 x}\right)^{1 / 2+3 x} \\
\quad=\sum_{k=1}^{\infty}\left(\frac{1}{k}-\frac{2 x}{k+1}\right)\left(z_{n}^{k}-z_{1}^{k}\right) \leq(2 x-1) \log \left(\frac{1}{1-z_{1}}\right) .
\end{aligned}
$$

On the other hand we have for all $n \geq 2$ and all $x>0$ :

$$
(1+n x)^{1 / n} \leq 1+x \quad \text { and } \quad 3 \gamma(x)-\gamma(3 x)-\left(1+\frac{2}{n}\right) \gamma(n x)+\gamma((n+2) x) \geq 0 .
$$


Therefore it suffices to prove that for all $\frac{1}{2} \leq x \leq 1$ :

$$
\left(\frac{1+3 x}{1+x}\right)^{2 x-1} \leq \sqrt{2 \pi e}
$$

which follows from the fact that the left hand side is bounded by 2 . With some modifications the proof given above also yields for all $n \geq 3$ :

$$
\frac{\Gamma\left(1+\frac{n}{p}\right)^{1+2 / n}}{\Gamma\left(1+\frac{n+2}{p}\right)} \leq \frac{\Gamma\left(1+\frac{2}{p}\right)^{2}}{\Gamma\left(1+\frac{4}{p}\right)} .
$$

Hence we have the following

Proposition 3.1. For all hyperplanes $H$ containing the origin, all $1<p<2$ and all $n \geq 2$ we have:

$$
\operatorname{Vol}_{n-1}\left(r(n, p) B_{p}^{n} \cap H\right) \geq \sqrt{\frac{\Gamma\left(1+\frac{4}{p}\right) \Gamma\left(1+\frac{1}{p}\right)^{3}}{\Gamma\left(1+\frac{2}{p}\right)^{2} \Gamma\left(1+\frac{3}{p}\right)}} \geq 1 .
$$

\section{REFERENCES}

[B1] K. Ball, Logarithmically Concave Functions and Sections of Convex Sets in $\mathbf{R}^{n}$, Studia Math. 88 (1988), 69-84. MR 89e:52002

[B2] K. Ball, Normed Spaces with a weak Gordon Lewis Property, Proc. Funct. Anal., Univ. of Texas and Austin, 1987-1989, Springer LNM 1470, 36-47. MR 93e:46013

[C] K. L. Chung, A Course in Probability Theory, Academic Press, 1974. MR 49:11579

[MeP] M. Meyer and A. Pajor, Sections of the Unit Ball of $\ell_{p}^{n}$, J. Funct. Anal. 80 (1988), 109-123. MR 89h:52010

[MiP] V. D. Milman and A. Pajor, Isotropic Position, Inertia Ellipsoids and Zonoids of the Unit Ball of an n-dimensional Normed Space, GAFA Seminar 87-89, Springer LNM 1376, 64-104. MR 90g:52003

[SS] G. Schechtman and M. Schmuckenschläger, Another Remark on the Volume of the Intersection of Two $L_{p}^{n}$ Balls, GAFA 89/90, 174-178, Springer 1991. MR 92j:52008

Weizmann Institute of Science, Rehovot, Israel

Mathematisches Seminar, Universität Kiel, Germany

InStitut FÜr Mathematik, Universität LinZ, Austria

E-mail address: schmucki@caddo.bayou.uni-linz.ac.at

Current address: Institut für Mathematik, J. Kepler Universität, A-4040 Linz, Austria 